\title{
A Historical Perspective to Fabrication in Architecture for Preserving Heritage
}

\author{
Mine Özkar ${ }^{1}$, Begüm Hamzaoğlu², Sibel Yasemin Özgan ${ }^{3}$ \\ ${ }^{1,2}$ Istanbul Technical University ${ }^{3}$ MEF University \\ 1,2\{ozkar|hamzaoglu\}@itu.edu.tr3ozgans@mef.edu.tr
}

\begin{abstract}
Digital technologies have recently been at the forefront of the causal link between making and design. A growing number of architecture programs of universities incorporates fabrication to the educational environment, and even to the curriculum. Fabrication technology is now considered among the set of tools students are expected to acquire a basic knowledge of and skills in. Nevertheless, the pedagogical potential of fabrication in communicating traditions of making is underused in an oversight of the continuity of the relevant know-how. Our position is that traditions of making can be the subject matter of fabrication with the objective to remedy the role of fabrication tools in architectural history, sustainable architectural production, and in the field of digital heritage. In this paper, we report on two comparative studies that illustrate how the instrumental factors of two historical crafts can be articulated using fabrication.
\end{abstract}

Keywords: computational design, craft, stone carving, tile mosaic

\section{INTRODUCTION}

Whereas the building industry is mostly driven by economic forces, architecture is amiably considered a constructional craft and culture (Frampton, 1995). There is a good number of crafty architectural practices and institutions of architectural education that promote hands-on and 1:1 explorations where the "poetics of construction" are in the foreground. However, the fabrication efforts that involve state-of-theart tools in educational environments are more on the technology side of things and do not always support the poetics or the traditions of making. Although there is an increasing number of specialized studios for improving fabrication skills, most of the learning involves the rote use of the available technology. Whereas the traditions of making in architecture education call for a greater contact with histor- ical methods of construction, these efforts fall short in serving the connections between the past and present ways of architectural making.

Almost in all schools with the sufficient economic resources, site models, and sometimes architectural models, are assembled of laser-cut pieces of sheet materials. Computer generated data translates to tangible objects and contours of tangible objects can be captured to produce detailed digital models and numeric data (Dunn, 2012). CNC routers are popular for producing models of more plastic forms whereas prototyping is reserved for detailed productions with more flexible time frames. Celani (2012) already offers an initial assessment of the pedagogical impacts of digital fabrication. Our position is from the viewpoint of architectural heritage and how traditions of making can be the subject matter of fabri- 

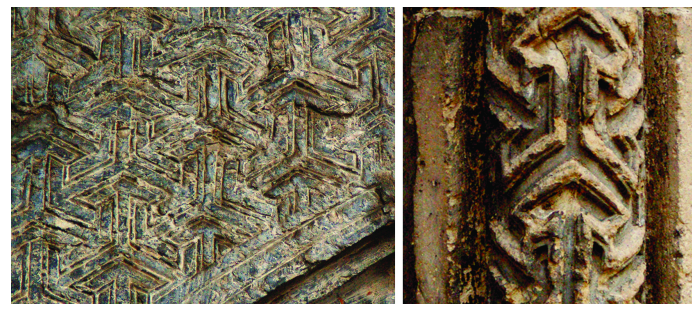

cation. In this light, it is important to make use of digital fabrication not just as celebrated new technology but also in connection with the history of making, i.e. using the new tools to better understand some past knowledge of making. Our broader study aims to remedy the role of fabrication tools in architectural history, in sustainable architectural productions, and in the field of digital heritage. This paper reports two studies that illustrate the instrumental factors of two historical crafts as articulated through fabrication.

\section{FABRICATION WITH HISTORICAL REFER- ENCES}

Fabrication tools are the state-of-the-art technology that bind making and design. Yet, this link is not always explored from a historical standpoint. A few exceptions are studies that explore the history of fabrication tools in reference to prominent examples of construction of expressive forms (Burry, 2012). Moreover in the recent years, the field of architectural heritage has seen an increased interest in the technologies, and digital fabrication tools are becoming common to produce physical reproductions (Scopigno et al., 2017). Nonetheless, these applications are limited to the reproduction of form and do not incorporate the knowledge from the original constructional process. However, preservation of heritage is reliant predominantly on this knowledge. The fabrication tool produces a simulation of the form but it does not always simulate the original tool nor the process that had produced the form in its original time and place. Recent work that considers the potential of fabrication in sustaining traditions of architectural making

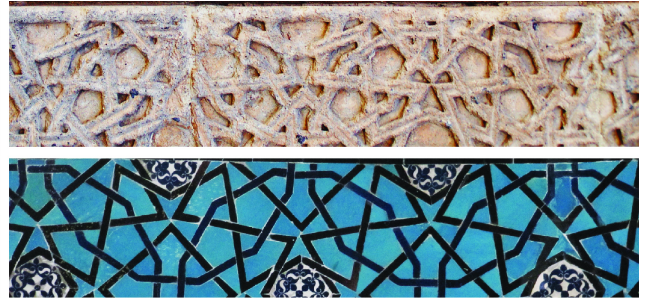

are promising. Earlier, Klinger (2001) had drawn attention to looking at historic expressive architectural form through the viewpoint of fabrication to examine new fabrication techniques. Villalon et al. (2009) had worked on robotic brick laying that looks at historical examples for exercising knowledge of construction, materials and cost. And more recently, Martins and Sousa (2014) have been exploring longestablished materials through digital fabrication with the purpose of sustaining traditional know-how.

Acknowledging the potential of current tools in studying the constructive techniques of architectural heritage, we explore two historical techniques from medieval Anatolia. We worked with volunteers in the format of a charette. The participants were mostly second year architecture students and novices in design technology and historic construction techniques. The exception was two graduate students who were highly experienced in architectural design computing and had some knowledge of the historic construction techniques considered in the study.

We provided the participants with two existing patterns representative of the geometric ornamentation common to monumental architecture of the Seljuk era (Figure 1). The first design (Pattern 1) has a three-fold symmetry. It is carved in two instances on flat and curved stone surfaces in the Bimarhane of Amasya. The second design (Pattern 2) has a fourfold symmetry. It is applied, again in two instances, in the Sahip Ata Mosque in Konya. One instance is carved on the exterior stone surface, and the other is assembled as tile mosaic on an interior panel. Patterns of different symmetry groups and that they underwent adaptations with different techniques were
Figure 1

Pair on the left -

Pattern 1 from

Amasya. Pair on the

right - Pattern 2

from Konya

(Photographs by

Ezgi Baştuğ and

Sibel Yasemin

Özgan) 
Figure 2

The making rules and corresponding phases of the subtraction for the two existing versions of Pattern 1. The center photo and the far right photo show the finished products.
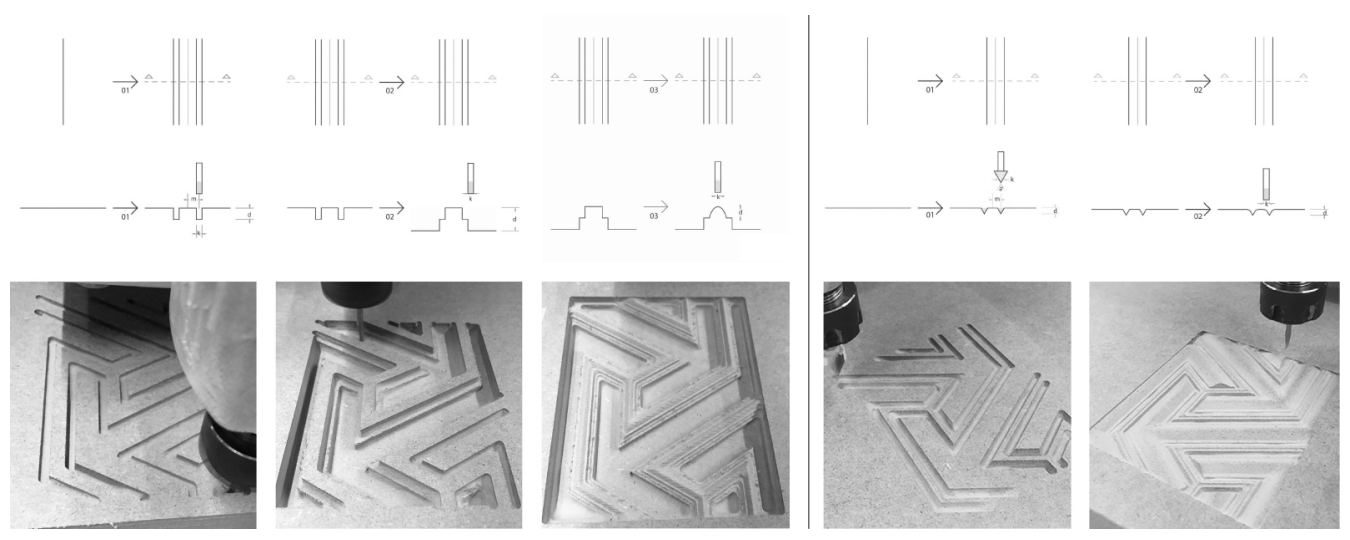
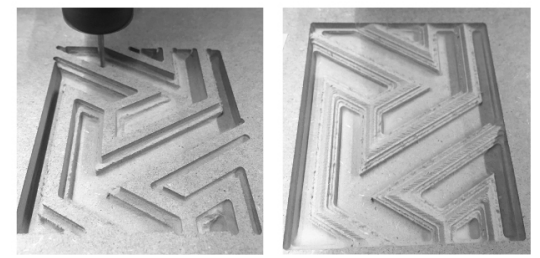

comparison to the basic unit of the pattern and the curve does not have a considerable impact on the outcome. Due to the limitations of the axes of movement, the router cuts the areas near the two edges of the panel in a tapered fashion. This results in distorted design elements and impact the homogeneity of the pattern towards the tapered edges of the single-curved surface (Figure 3).

For models 01-05, v-bit cutters are used to carve out the closed polylines. For models 01-03, the cutter has a 30 degrees taper and the depth is the only parameter that is changed. The results demonstrate the contrasting effect of the deeper cuts. For models 04 and 05 , the depth is kept constant but the cutter taper angles vary ( 30 and 45 degrees respectively). The depth in model 04 results in broader tapered surfaces and diminished flat top surfaces. In model 05 , due to the 45 degree taper of the cutter, there are almost no flat top surfaces left.

The group of participants who produced models 06-09 with Pattern 2, chose to carve out the lines rather than the polygonal surfaces these bound. This choice and that they used only the flat mill resulted in two-dimensional outcomes. The group experimented with varying the depth of the cut across the panel and the sequences of the tool path to alter the background foreground relations. Especially in model 09, a rotating motif, different than the sym- 


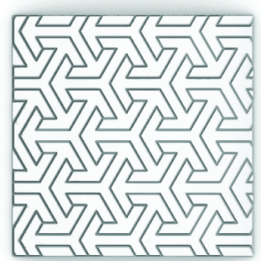

01

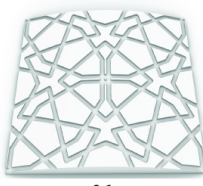

06

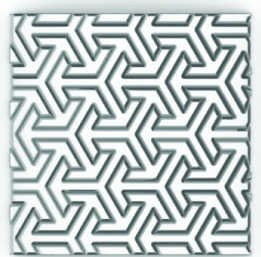

02

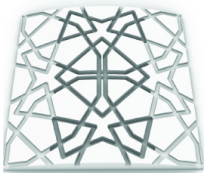

07

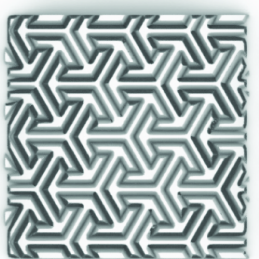

03

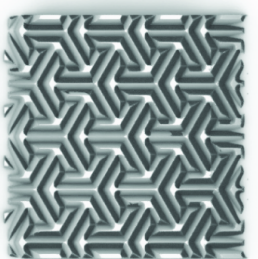

04

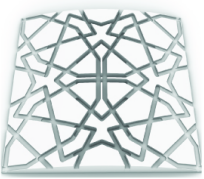

08

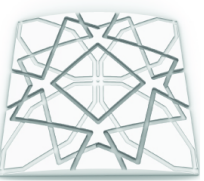

09

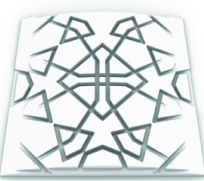

10

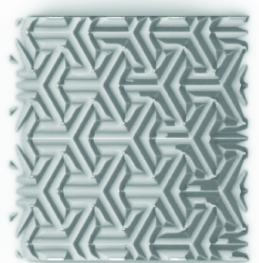

05

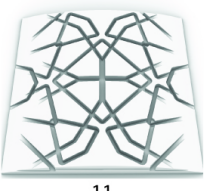

11 metrical original design, and a new weaving pattern emerge.

The third group that produced models 10 and 11 , worked similarly to the second group. Applying Pattern 2, they use the same flat mill and keep the depth at a constant $2 \mathrm{~mm}$. Instead of engraving the parts in the base geometry as separate lines, they define particular shapes as closed polylines. Then they assign the tool path to follow either the inside or outside of these closed shapes. The offset distance from the base geometry is kept at a constant. This tool path design results in diminished polygonal areas and conjoined lines, yielding a pattern different than the original, to the extent that the square in the center of the motif in model 11 is completely eliminated.

The results showed significance in how the original visual design changed through just a few and slight deviations in the application of the variables. The study attests to the role that the cutting tool and other parameters of making play in the creation of the visual, spatial, and structural effect. The participants also gave feedback on the awareness they acquired with regards to both the historical and contemporary crafting of what is usually perceived as a straightforward cutting of a traditional two dimensional design.

The second set of cases involves a different mate- rial and its relevant techniques and variables. Different than the subtractive processes of stone carving, the material and technique selected to serve as reference to this second set is additive and is historically known as the tile mosaic. In the historical technique, small shapes are cut from plain or glazed tiles and, following the design, assembled as panels which are then adhered to architectural surfaces. In the study, the application of Pattern 2 involves the cutting of the tile mosaic pieces according to an understanding of the design and the tessellation of the mosaic pieces on differently curved surfaces. The variables this time are based on the geometric features of the application surface, the symmetry properties of the design, and the division of the design into two levels of primitives, namely the buildable panels and the mosaics.

\begin{tabular}{|c|c|c|c|c|c|c|}
\hline & & & TOO & & TOOL & _PATH \\
\hline Model & Pattern & Shape & Angle & Dia $(\mathrm{mm})$ & Base Geom. & Depth $(\mathrm{mm})$ \\
\hline 01 & 1 & V & 30 & 12.7 & L & 2 \\
\hline 02 & 1 & v & 30 & 12.7 & L & 4 \\
\hline 03 & 1 & v & 30 & 12.7 & $\mathrm{~L}$ & 6 \\
\hline 04 & 1 & v & 30 & 12.7 & L & 8 \\
\hline 05 & 1 & v & 45 & 12.7 & L & 8 \\
\hline 06 & 2 & Flat & - & 5 & L & 2 \\
\hline 07 & 2 & Flat & - & 5 & L & $2-8-2$ \\
\hline 08 & 2 & Flat & - & 5 & L & $2-4-8-4-2$ \\
\hline 09 & 2 & Flat & - & 5 & $\mathrm{CP}$ & $1-2-4$ \\
\hline 10 & 2 & Flat & - & 5 & $\mathrm{CP}$ & 2 \\
\hline 11 & 2 & Flat & - & 5 & $\mathrm{CP}$ & 2 \\
\hline
\end{tabular}

Figure 3

Top row - models 01 through 05 with Pattern 1. Bottom row - models 06 through 11 with Pattern 2.
Table 1

The parameters and values for the subtractive manufacturing models (CP - closed polylines, L - lines) 
Figure 4

Top row - division of the spherical surface into quads Bottom row - the flat panel in laser cutting and the finished product

Figure 5

An adaptation of the motifs to the slices of the spherical surface.
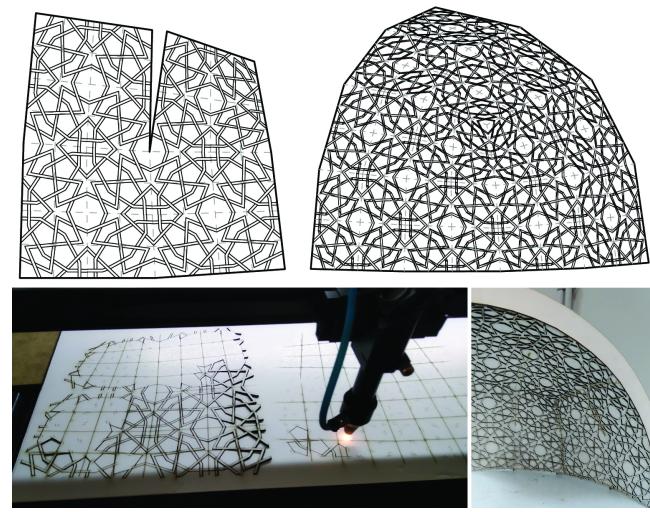

Two groups who worked on the tile mosaic cases applied Pattern 2. The task was for them to apply it on the interior surface of a hemispherical dome. The production means, in order to keep accord with the cut tiles of the historical designs, were specified as laser cutting sheet materials. The challenges were not only the adaptation of the four-fold pattern on the sphere but also achieving curved surfaces out of the flat pieces cut from planar sheets. The fourth group consisted of highly experienced design computation experts who were comfortable users of $3 d$ modeling tools. Seeing the advantage of the fourfold symmetry of the pattern design, they matched it to a quad faced sphere in a digital model. This did not offer an ideal match to the curve of the surface. At the degree of detail they worked in, one eighth of the sphere comprised of 12 quads. However this approach resonated with the knowledge the builders possibly had in the medieval era and could have easily applied. Manuscripts as early as from 10th century show mathematicians' instructions to craftsmen for dividing the sphere into polyhedra (Al-Buzjani, ca. 940-998).

After subdividing the spherical surface into irregular quads, and splitting the four-fold repeating pattern into matching quads, the group calculated the distances of the planar surface of the quads to the sphere. Based on the distance map, they were able to designate lines to cut the pattern in order to better fit the curve and compensate the distances. The shrinkage of the pattern elements on the wider surface of the sphere was negligible. The result shows distorted units of the pattern. The distortion is greater in the central area of the finished model as the quads were the most distorted there. Still, the overall application yields the desired continuity of the design. Differently, most original patterns on Islamic domes display altered motifs along the meridians to fit the tapering dome moving upwards.

The last group of participants took on the same task as above but divided the spherical surface into thin vertical slices. They also subdivided these slices into quads in an attempt to fit sections from the pattern onto individual slices. Thus, they faced a problem as parts of the pattern overlapped on narrow sections that are close to the spherical apex. Their solution was to manipulate some areas of the patterns so that the entire pattern could match the surface segments. At the end, shapes that do not belong to the original pattern emerge. Although the overall governance of the spherical geometry was not very successful, this group was resourceful in similar ways to their historical counterparts who, as mentioned above, adapted the patterns to fit in tapered upper levels of the dome. Nevertheless, it was not possible in this approach to practically and systematically produce tile mosaics by cutting with the laser and assembling in quad panels.

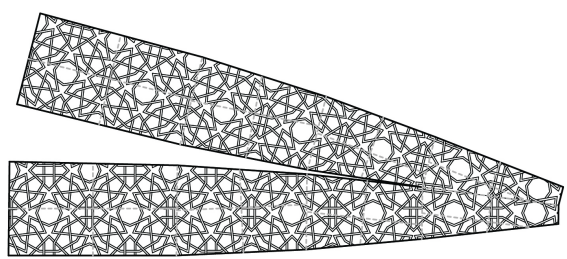

Results corroborate the early proposition that designs transform with reference to the tool or the variables and conditions of their making. These results are significant in outlining for these cases the articulations of the physical limitations and opportunities the original traditional methods offered, and 
how they can be controlled, improved, and taught in depth using current technologies at hand.

\section{CONCLUSION}

For the subtractive manufacturing of geometric patterns, the instrumental factors are the shape and size of the cutting tools, the paths designed for the tool to travel, and the depth of the cuts. These respectively correspond to the tools used by the craftsmen and how they shaped the cut, the order of cuts they performed on the stone, and how deep they cut into the stone. The speed of the cutting is yet another factor but is not explored within the scope of this study. Previous observations show that the speed has impact on the textures created with the cuts. As all these factors correspond to aspects of the historical technique, the exploration provides the students with knowledge on both the contemporary tools and the historical methods of making. Craftsmanship is conceivable with control over these parameters.

For the design and execution of the additive manufacturing of geometric patterns with reference to the tile mosaic technique, the instrumental factors are the degree of the curvature of the applied surface, the symmetry group in the pattern, the scale of the basic motif of the pattern with regards to the size of the applied surface, and the adaptation of the pattern to the curve in increments through the design of smaller panels.

In concert with the growingly popular global history of architecture, local building cultures that were previously abbreviated or entirely neglected in survey courses of architecture schools' curricula, have now gained recognition. Especially in societies which, despite following the global market rules, are still laden with traditional values, the recognition is both intellectually and practically important. In local cultures where designer-builders' knowledge is passed on to the next generations tacitly or through non-abstract means, modernist perspectives to the preservation of building heritage are questionable. Moreover, in architectural preservation, a field with powerful influence over memory and narrative, the case of constructional form is often incapacitated by regulations, and at the same time, distanced from contemporary design research that has the potential to cast new light on assumed ontologies of architecture and limited depictions of past design cultures.

\section{ACKNOWLEDGMENT}

Authors wholeheartedly thank all the participants and extend further gratitude to Fatih Uzun and Demircan Taş for taking on the research challenge.

\section{REFERENCES}

Al-Buzjani, Abu'l-Wafa ca. 940-998, Kitab Fi ma yahtaj ilayhi al-sani' min al-a'mal al-handasiyya (On the Geometric Constructions Necessary for the Artisan), Istanbul, Suleymaniye Library, Ms. Ayasofya 2753

Bonner, J 2017, Islamic geometric patterns: Their historical development and traditional methods of construction, Springer, New York

Burry, M 2012, 'The Persistence of Faith in the Intangible Model', in Ayres, P (eds) 2012, Persistent Modelling: extending the role of architectural representation, Routledge, London and New York, pp. 26-40

Celani, G 2012, 'Digital Fabrication Laboratories: Pedagogy and Impacts on Architectural Education', Nexus Network Journal, 14(3), pp. 469-482

Dunn, N 2012, Digital fabrication in architecture, Laurence King, London

Frampton, K 1995, Studies in Tectonic Culture: The Poetics of Construction in Nineteenth and Twentieth Century Architecture, The MIT Press, Cambridge, MA

Klinger, KR 2001 'Making Digital Architecture: Historical, Formal, and Structural Implications of Computer Controlled Fabrication and Expressive Form', Proceedings of eCAADe 2001, Helsinki, Finland

Martins, PF and Sousa, JP 2014 'Digital Fabrication Technology in Concrete Architecture', Proceedings of eCAADe 2014, Newcastle upon Tyne, UK, pp. 475484

Scopigno, R, Cignoni, P, Pietroni, N, Callieri, M and Dellepiane, M 2017, 'Digital Fabrication Techniques for Cultural Heritage: A Survey', Computer Graphics Forum, 36, pp. 6-21

Villalon, R, Lieberman, $\mathrm{H}$ and Sass, L 2009'Breaking down brick walls: design, construction, and prototype fabrication knowledge in architecture', $\mathrm{CHI}$ '09 Extended Abstracts on Human Factors in Computing Systems, ACM, New York, NY, USA, pp. 4261-4266 